\title{
Functionally Oriented Alignment of the Lower Extremity Reflecting the Direction of Gait for Healthy Elderly, Knee Osteoarthritis, and Total Knee Arthroplasty Subjects
}

\author{
Takashi Sato $^{1} \cdot$ Tomoharu Mochizuki $^{2}$ [D $\cdot$ Ryota Katsumi $^{1} \cdot$ Yuki Takahashi $^{1}$
}

Received: 23 May 2020 / Accepted: 21 September 2020

(c) The Author(s) 2021, corrected publication 2021

\begin{abstract}
Purpose The objective of this study was to evaluate the functional lower extremity alignment based on both position and motion [functionally oriented alignment (FOA)] of the knee in healthy elderly, varus osteoarthritis (OA), and total knee arthroplasty (TKA) subjects.

Methods This study evaluated 87 knees in 24 healthy elderly ( $72 \pm 5$ years), 39 varus OA ( $72 \pm 6$ years), and 24 TKA ( $75 \pm 4$ years) subjects. A 3D assessment system was used on 3D models and biplanar long-leg radiographs with the toe angle reflecting gait direction, by applying a 3D-to-2D image registration technique. In the world coordinate system, the $y$-, $z-$, and $x$-axes were defined as gait direction, gravity direction, and the cross product of $y$ - and $z$-axes, respectively. The parameters were: (a) coronal inclination, sagittal inclination, and transverse direction of the femur and tibia relative to the ground and (b) the difference between the $y z$-plane of the world coordinate system (functional plane) and the $y z$-plane of the femoral or tibial coordinate system (anatomical plane).

Results The femur had more medial and posterior inclination and the tibia had more lateral and anterior inclination in osteoarthritic knees as compared to healthy knees, and TKA knees had inclinations similar to healthy knees. Rotation was similar or different in the anatomical and functional planes among the subjects with the healthy, knee OA, and TKA.

Conclusions The association between the anatomical and functional planes and the position of each bone relative to gravity varied depending on the condition of the knees.

Level of Evidence: Level of III
\end{abstract}

Keywords Functionally oriented alignment $\cdot$ Lower extremity alignment $\cdot$ Knee forward $\cdot$ Gait direction $\cdot$ Bone inclination . Functional plane $\cdot$ Anatomical plane

Tomoharu Mochizuki

tomohana@med.niigata-u.ac.jp

Takashi Sato

takuukat2032@gmail.com

Ryota Katsumi

r-katsumi08@live.jp

Yuki Takahashi

titaneggman@yahoo.co.jp

1 Department of Orthopaedic Surgery, Niigata Medical Center, 3-27-11, Kobari Nishi-ku, Niigata 950-2022, Japan

2 Division of Orthopedic Surgery, Department of Regenerative and Transplant Medicine, Niigata University Graduate School of Medical and Dental Science, 1-757 Asahimachi-dori Chuo-ku, Niigata City, Niigata 951-8510, Japan

\section{Background}

Lower extremity alignment is a critical factor that explains issues related to the onset and progression of knee osteoarthritis (OA) [1-5]. These factors are also important with respect to clinical results and prosthesis longevity in total knee arthroplasty (TKA) [6-8].

Lower extremity alignment is based on an anatomical coordinate system [anatomically oriented alignment (AOA)] [9-11] that defines the direction of anteroposterior plane, vertical to the extension-flexion axis of the knee [12-14] as "knee forward" (Fig. 1). For example, in the femoral or tibial coordinate system, the anteroposterior anatomical plane, meaning "knee forward", was generally defined as the sagittal plane in the femoral or tibial coordinate systems, respectively $[15,16]$. At present, it remains unclear whether 
Fig. 1 Anatomical and functional planes. The anteroposterior plane ( $Y$-Zf plane) vertical to the extension-flexion axis of the femur was defined as "knee forward". The difference between the anatomical and functional planes indicated the difference between the "knee forward" and the gait direction. In this study, the anatomical plane was defined as each of the $y z$-plane of the femoral and tibial coordinate system, respectively. The functional plane was the direction of the $y z$-plane of the world coordinate system. $Y w, Y f$, and $Y t=y$-axis of the world, femoral, and tibial coordinate system; $Y-Z \mathrm{w}, Y$-Zf, and $Y-Z \mathrm{t}=y-\mathrm{z}$ plane of the world, femoral, and tibial coordinate system

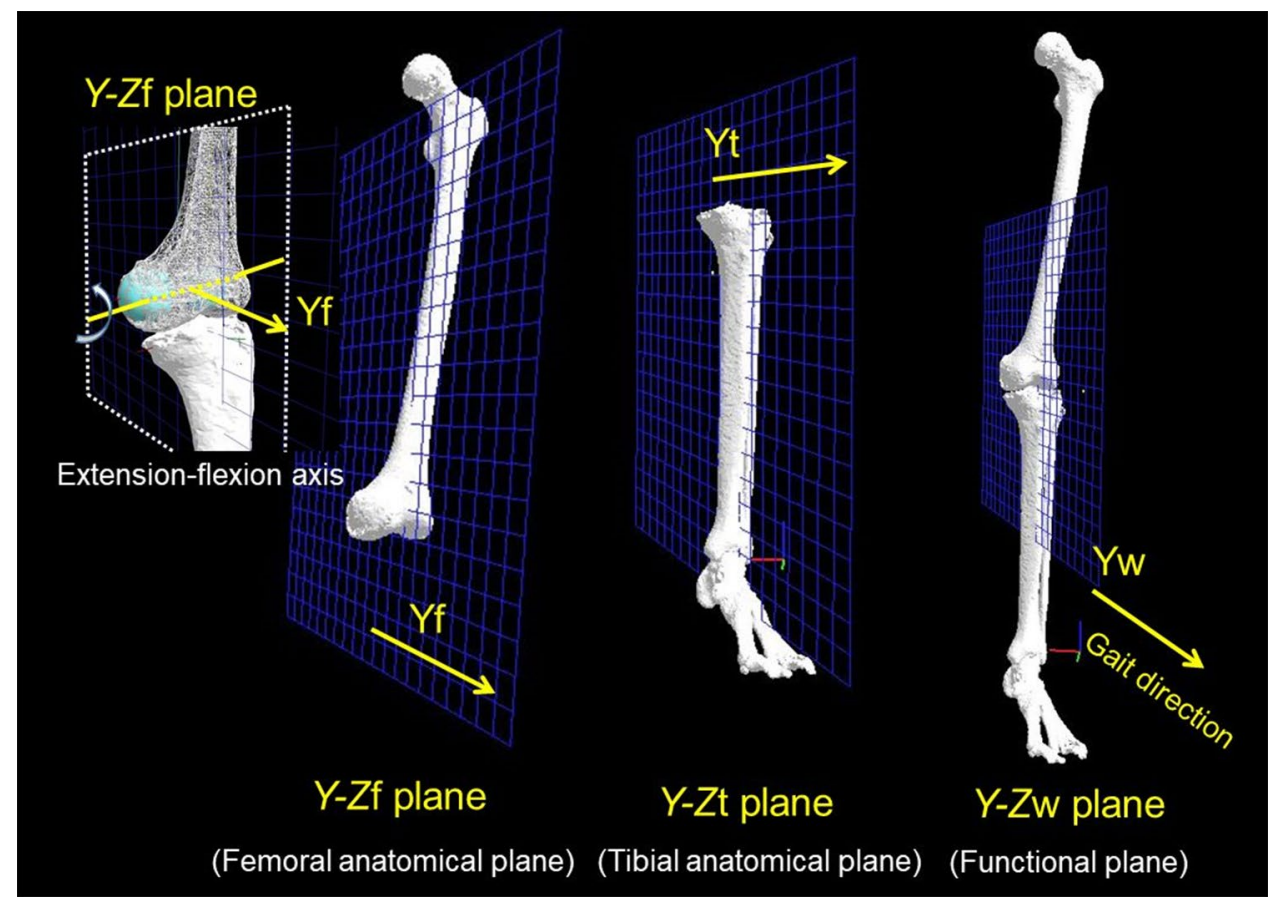

"knee forward" is femoral or tibial anteroposterior anatomical plane in healthy knees.

For healthy knees, either the femoral or tibial anteroposterior anatomical plane assumingly corresponds to the gait direction. However, in individuals with knee OA, anatomical plane may not correspond to the gait direction due to torsional deformities of the bone and/or rotational mismatch between the femur and tibia [17-19]. Previous reports documented the association of external rotation gait with osteoarthritic knees [20, 21], a possible example of discordance between the anteroposterior anatomical plane and the gait direction. As regards to TKA, the authors have occasionally experienced inconsistency between the gait direction and the anteroposterior axis of the implant, even when the common X-ray in knee forward view is showing acceptable implant position relative to the bone. The different relationship between the two planes, compared to what has been observed for healthy knees, may cause eccentricity and overloading at the knee joint ultimately leading to further progression of knee $\mathrm{OA}$ and the persistence of serious problems after TKA [22, 23].

As such, use of AOA as the sole means of evaluation for the lower extremity alignment is not sufficient; a means for the evaluation of functional alignments based on both position and motion [functionally oriented alignment (FOA)] will be necessary for a full assessment of joint dysfunction. When humans walk in a forward direction, the functional plane defining the pelvis, hip, and knee joints coincides $[17,18,24]$; extensor mechanism, such as the quadriceps femoris, is assumingly maintained in the forward position as the gait direction. In other words, the gait direction can reflect the function of the lower extremity, such as extensor mechanism. As the toe angle can be related to the tibial torsion, the toe angle itself may not necessarily coincide with the gait direction; the gait direction is presumed to better reflect the function of the lower extremity than the toe angle. Therefore, in this study, FOA can be evaluated by a coordinate system that defines the gait direction, not the toe angle, as the " $y$-axis" and vertical direction to the ground as the "z-axis". The objectives of this study are: (a) to define the new parameter "FOA", (b) to evaluate FOA in elderly subjects with healthy knees to generate reference data, (c) to assess FOA in subjects with knee OA and those who have undergone TKA, and (d) to compare FOA with AOA in each subject. As far as clinical relevance is concerned, this study would demonstrate the potential to identify one of the causes of the onset and progression of knee OA and serious adverse problems after TKA.

\section{Materials and Methods}

A total of 107 elderly Japanese volunteers with no knee complaints and no history of joint disease or major injury in the lower extremity were prospectively reviewed. Physicians assessed their general and lower extremity conditions using physical tests and radiographs, and excluded seven subjects with radiographic evidence of knee OA. Of 100 healthy elderly patients (50 men, 50 women) with grades $0-1$ on the Kellgren-Lawrence $(\mathrm{K}-\mathrm{L})$ classification [25] and no radiographic knee OA $(\mathrm{K}-\mathrm{L} 0,1)$, the healthy volunteers were randomly selected $[3,17]$. For the subjects 
with advanced knee OA (K-L grade 3 or 4 ), the subjects with the preoperative TKA were randomly selected from the database in our institute. In TKA, the patients with the follow-up duration of over 1-year post-procedure were randomly selected from the database in our institute.

In this study (Table 1), we evaluated 24 knees from healthy elderly subjects ( 6 females and 6 males) with a mean $( \pm \mathrm{SD})$ age of $72 \pm 5$ years and mean body mass index (BMI) of $21.4 \pm 1.2 \mathrm{~kg} / \mathrm{m}^{2}$. We also evaluated 39 advanced osteoarthritic varus knees ( 20 females and 2 males with 35 and 4 knees, respectively); this group had a mean age of $72 \pm 6$ years and mean BMI of $25.8 \pm 3.6 \mathrm{~kg} / \mathrm{m}^{2}$. We also included 24 patients of postsurgical TKA (14 females and 1 male with 22 and 2 knees, respectively) with a mean age of $75 \pm 4$ years and mean BMI of $25.9 \pm 3.2 \mathrm{~kg} / \mathrm{m}^{2}$; the patients with history of TKA who were evaluated were at least 1 -year post-procedure. The $\mathrm{K}-\mathrm{L}$ grades showed $\mathrm{K}-\mathrm{L} 0$ (8 knees) or K-L1 (16 knees) in healthy knees and K-L3 (4 knees) or K-L4 (35 knees) in knee OA, respectively.

All TKAs were performed using the Evolution ${ }^{\circledR}$ Medial Pivot Knee system (MicroPort Orthopedics Inc., Arlington, TN, USA) with cruciate substituting inserts. The surgical procedure included resection of the posterior cruciate ligament and the femoral distal surface was cut perpendicularly to the mechanical axis in the coronal plane. The sagittal alignment was adjusted to prevent anterior notch in the femur and the rotational alignment of the femoral component was set parallel to the surgical epicondylar axis [19]. The tibial posterior slope was $3^{\circ}$; the proximal tibia in the coronal plane was cut at $90^{\circ}$ to the mechanical axis of the tibia. The rotational position of the tibia was based on the Akagi line [26]. The patella was not resurfaced and lateral retinaculum release was not performed.

\subsection{Outline of a Three-Dimensional Lower Extremity Alignment Assessment System}

The unique three-dimensional (3D) lower extremity alignment assessment system (Knee CAS; LEXI, Inc., Tokyo, Japan) was used as earlier described [4, 5, 8-11, 15-19, 27-36] to evaluate FOA parameters. 3D digital models of the femur and tibia were reconstructed from computerized tomography (CT) images (Canon Aquilion 64 CT, Canon Medical Systems, Tochigi, Japan) using 3D visualization and modeling software (ZedView; LEXI Inc., Tokyo, Japan). Biplanar radiographs of the entire lower extremity were obtained in weight-bearing conditions. The subject stood on the turn-stage, and the entire lower extremity was imaged in the frontal and $60^{\circ}$ oblique directions with computed radiography. The 3D digital bone and component models were projected on biplanar radiographs by a 3D-to-2D image registration technique (Fig. 2). All alignments and deformities were calculated automatically. As regards to accuracy, our previous study reported that median values of the absolute error in estimating relative positions were within $0.5 \mathrm{~mm}$ and $0.6^{\circ}$ for the femur with respect to the tibia [27].

\subsection{Standing Position in Biplanar Radiographs}

Biplanar radiographs of the entire lower extremity were obtained in weight-bearing conditions with the right and left toe angles, respectively, reflecting the gait direction. The gait direction overall and the toe angle relative to the gait direction is important for accurate measurement of FOA. These measurements to determine the gait direction and toe angles were performed using the following methodology (Fig. 3): two pins were attached to the anterior and posterior parts of the sole of the shoes worn by the test subjects which created perforations in paper laid out on the floor during free walking. The gait direction was determined over three cycles of free gait via assessment of the direction of the perforations made by the anterior pin. The average toe angles were determined by the gait direction and the direction of the perforations made by both the anterior and posterior pins. In the cases with bilateral sides, the toe angles were separately evaluated on the right and left sides for each of the subjects as toe angles on the right and left sides could differ in the same subject. The toe angles were presented as the positive angles in external rotation relative to the gait direction. The average $\pm \mathrm{SD}$ of the toe angles were $6.5 \pm 4.5^{\circ}$ (range, $-0.5-14.5^{\circ}$ ) in healthy knees, $1.9 \pm 7.1^{\circ}$

Table 1 Demographic data

\begin{tabular}{|c|c|c|c|c|c|c|c|}
\hline \multirow[t]{2}{*}{ Variables, mean or proportions } & \multicolumn{2}{|c|}{ Healthy $(n=24)$} & \multicolumn{2}{|c|}{ OA $(n=39)$} & \multicolumn{2}{|c|}{ TKA $(n=24)$} & \multirow[t]{2}{*}{$p$ value } \\
\hline & Mean & $95 \% \mathrm{CI}$ & Mean & $95 \% \mathrm{CI}$ & Mean & $95 \% \mathrm{CI}$ & \\
\hline Age, years & 72 & $70-74$ & 72 & $70-74$ & 75 & $73-77$ & $<0.001 *$ \\
\hline BMI, $\mathrm{kg} / \mathrm{m}^{2}$ & 21.4 & $20.9-21.8$ & 25.8 & $24.6-27.0$ & 25.9 & $24.5-27.3$ & $0.032 *$ \\
\hline Toe angle (external rotation, + ) & 6.5 & $4.6-8.4$ & 1.9 & $-0.4-4.2$ & 2.1 & $-1.3-5.5$ & $0.025^{*}$ \\
\hline Sex (male:female), $n$ & $12: 12$ & & $4: 35$ & & $2: 22$ & & $<0.001^{*}$ \\
\hline
\end{tabular}

$B M I$ Body mass index; Toe angle was defined as the positive values in external rotation relative to the gait direction, $n$ knees

*Significant difference $=p<0.05$, n.s. $=p>0.05$ 


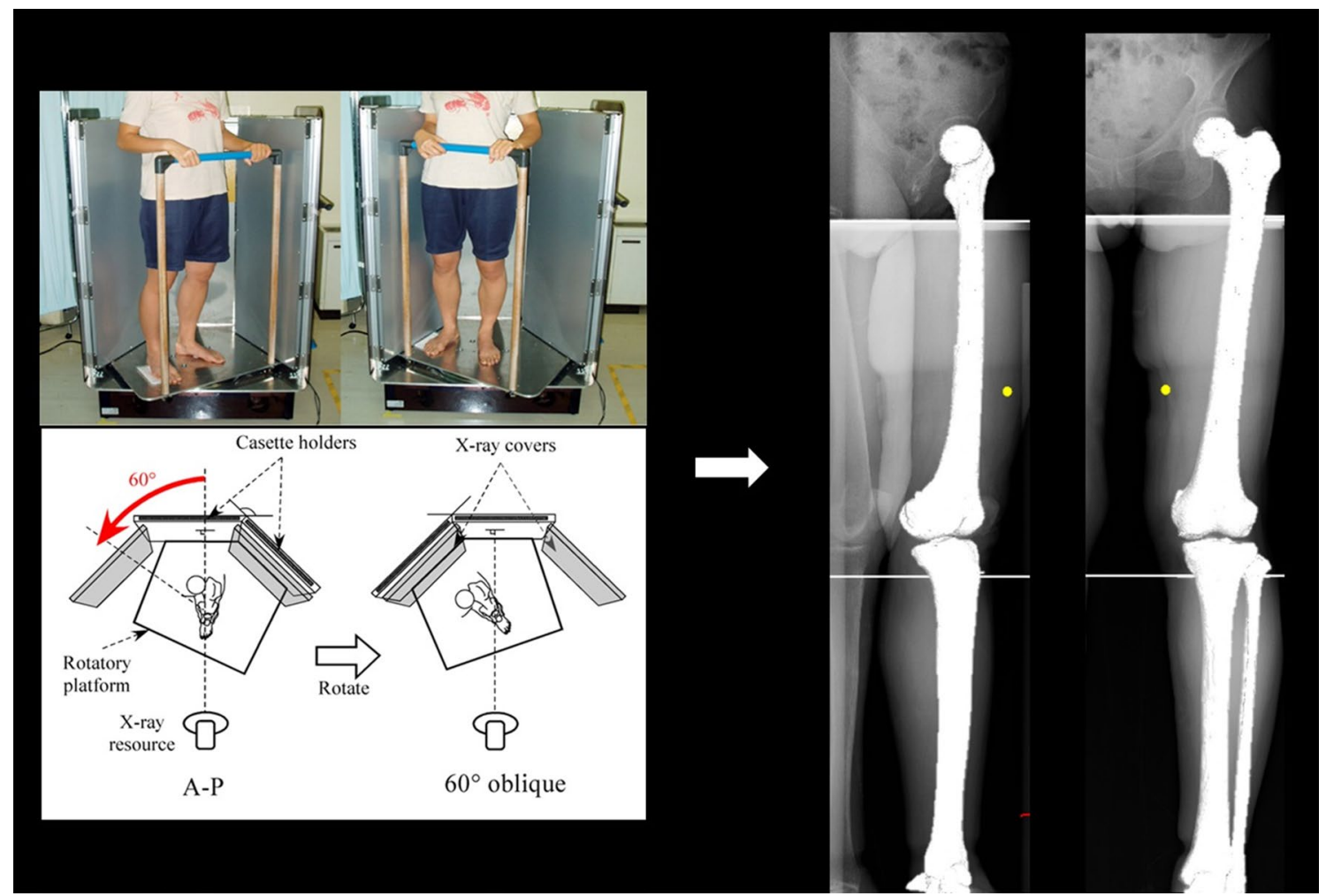

Fig. 2 Biplanar X-ray images of the entire lower extremity and 3D-to2D image registration technique. Biplanar X-ray images of the entire lower extremity were obtained under weight-bearing conditions. The

(range, $-17.6-20.0^{\circ}$ ) in knee OA, and $2.1 \pm 8.1^{\circ}$ (range, $-10.0-25.2^{\circ}$ ) in TKA (Table 1).

\subsection{Coordinate System}

With respect to the world coordinate system, the $y$-axis was defined as the gait direction, the $z$-axis was defined in the direction of gravity, and the $x$-axis was defined as the crossproduct between the $y$ - and $z$-axes. The anatomical femoral and tibial coordinate systems were established in reference to bony landmarks as defined earlier [15, 16] (Fig. 4).

\subsection{Assessment Parameters}

All the parameters used for the assessment of FOA were evaluated using the world coordinate system in weightbearing conditions that include (Figs. 5, 6) (a) coronal inclination, i.e., the inclination of the functional axes of the femur and tibia with respect to the coronal plane; (b) sagittal inclination, i.e., the inclination of the functional axes of the femur and tibia with respect to the sagittal plane; (c) transverse direction, i.e., the angle between the gait direction and the surgical epicondylar axis of the femur or the Akagi line of the tibia; (d) the coronal and sagittal alignments in
3D digital bone and component models were projected on biplanar $\mathrm{X}$-ray images by a $3 \mathrm{D}$-to-2D image registration technique

hip-knee-ankle angle (HKA) and rotational alignment in FOA and AOA; and (e) the difference between the anatomical and functional planes in both the femur and tibia.

The coronal inclination was defined as follows: the femoral functional axis is the line between the femoral head and the origin point of the femoral coordinate system. The tibial functional axis is the $z$-axis of the tibial coordinate system. The femoral and tibial coronal inclination is defined as the angle of each functional axis relative to the $z$-axis of the world coordinate system in the coronal plane, respectively (medial inclination: + ). The femoral and tibial sagittal inclination is defined as the angle of each functional axis relative to the $z$-axis of the world coordinate system in the sagittal plane, respectively (anterior inclination: + ). In the transverse plane, the surgical epicondylar axis is defined as the line connecting the lateral epicondylar prominence and the lowest point of the medial sulcus of the medial epicondyle. The Akagi line is defined as the line between the center of the posterior cruciate ligament attachment and medial edge of the tibial tuberosity. The transverse direction is defined as: (a) the angle of the surgical epicondylar axis relative to the $x$-axis of the world coordinate system on the femoral side (this angle means the same angle between the perpendicular 
Fig. 3 Gait direction and toe angle. The toe angle and gait direction were determined using the following methodology: two pins were attached to the anterior and posterior parts of the sole of the shoes worn by the test subjects which created perforations in paper laid out on the floor during free walking. The gait direction was determined over three cycles of free gait via assessment of the direction of the perforations made by the anterior pin. The average toe angle was determined by the gait direction and the direction of the perforations made by both the anterior and posterior pins. Biplanar radiographs of the entire lower extremity were obtained under weight-bearing conditions with the toe angle reflecting gait direction

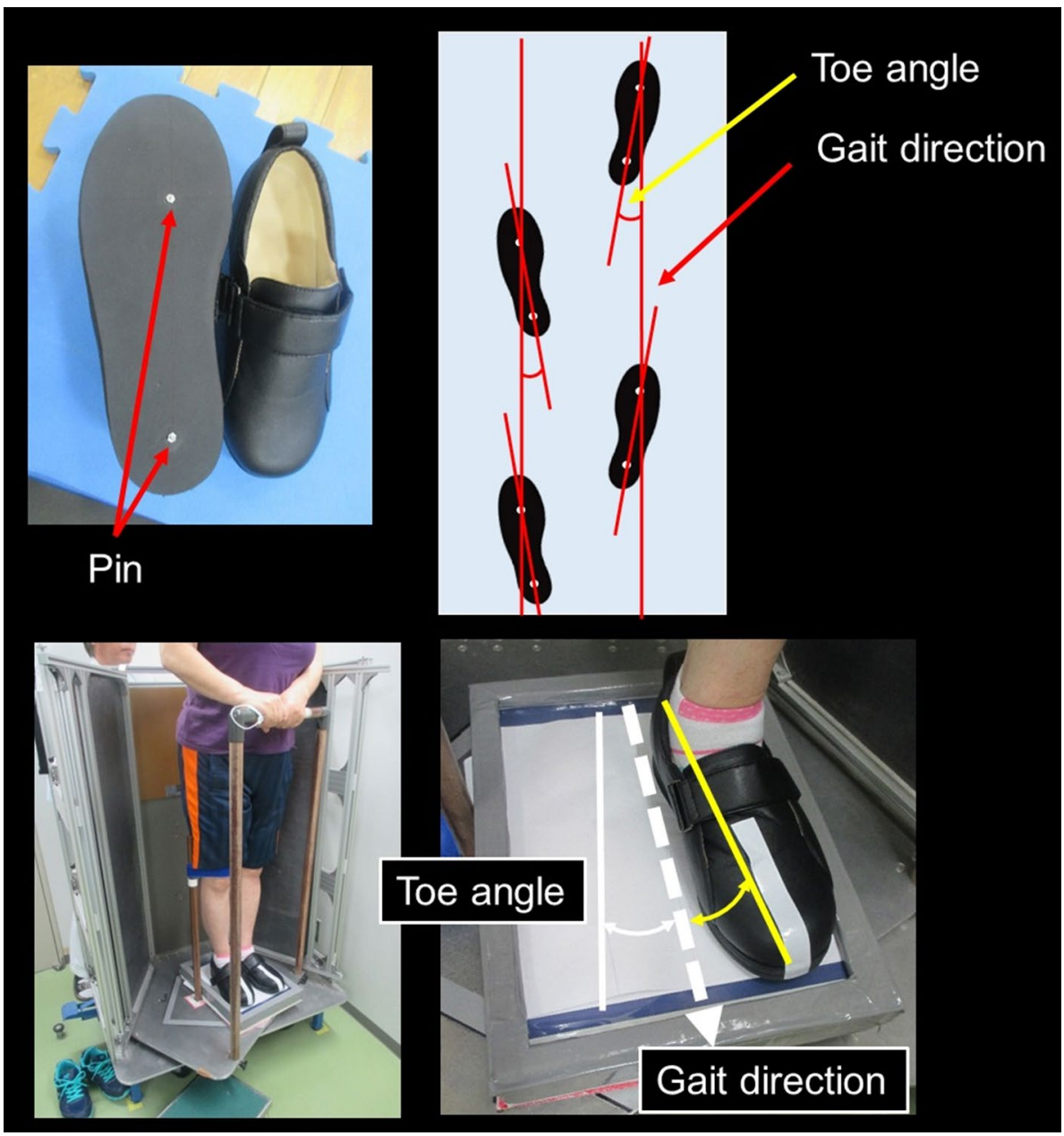

line to the surgical epicondylar axis and the $y$-axis of the world coordinate system) and (b) the angle of Akagi line relative to the $y$-axis of the world coordinate system on the tibial side, respectively (external rotation: + ). The coronal alignment in HKA was defined as the angle between the functional axes of the femur and tibia in the coronal plane of the world coordinate system (larger: varus). The sagittal alignment in HKA is defined as the angle between the functional axes of the femur and tibia in the sagittal plane of the world coordinate system (flexion: -). The rotational alignment is defined as the angle between the surgical epicondylar axis and the Akagi line in the world coordinate system (the tibial rotation relative to the femur; tibial external rotation: + ). In AOA, each of alignment is evaluated in the femoral coordinate system.

The difference between the anatomical and functional planes in the femur is defined as the angle of the $x$-axis in the femoral coordinate system relative to the $x$-axis in the world coordinate system (external rotation: + ). In the tibia, the two plane difference is defined as the angle of the $y$-axis in the tibial coordinate system relative to the $y$-axis in the world coordinate system (external rotation: + ).
As a simple interpretation of these parameters, the coronal and sagittal inclination represents the inclination of each bone relative to the ground, and the rotational parameters represent the rotational deviation of each bone with respect to the gait direction in FOA. Overall, the difference between the anatomical and functional planes reveals the deviation between "knee forward" and the gait direction. In this study, the anatomical plane is defined as the $y z$ plane in each of the femoral and tibial coordinate systems, respectively. The functional plane is the direction of the $y z$-plane in the world coordinate system.

This study was performed using a protocol approved by the institutional review board of our institution.

\subsection{Statistical Analysis}

Normality of the data was examined by using the Shapiro-Wilk test. The differences in these parameters among three groups (healthy, OA, and TKA) were statistically examined using the one-way analysis of variance with the post-hoc test (Tukey) when the data had a normal distribution, or the Kruskal-Wallis test with the post-hoc test 


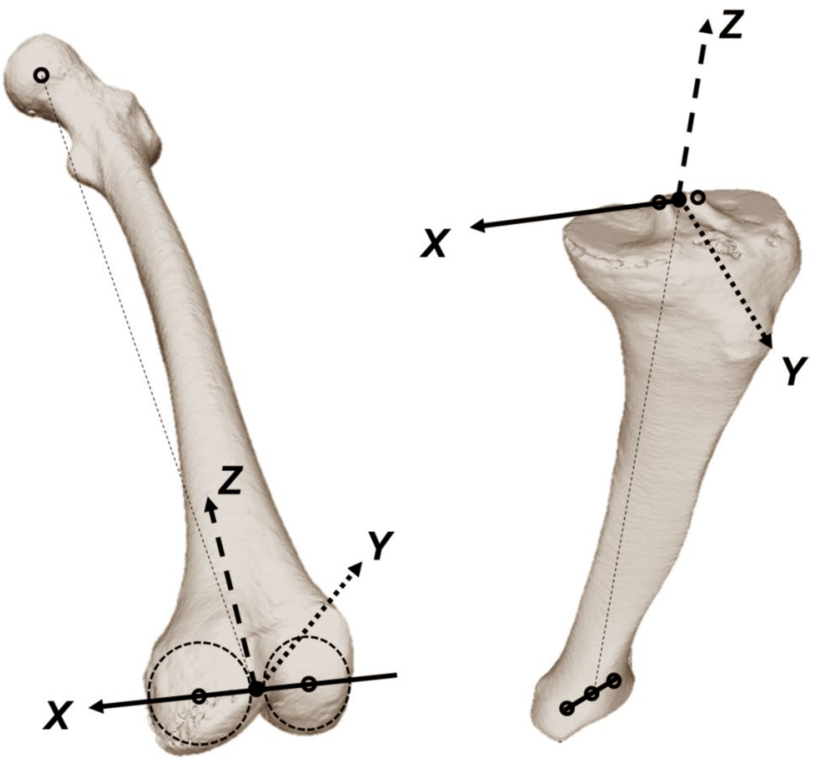

Fig. 4 Anatomical coordinate system of the femoral and tibial coordinate system. In the femur, the geometric center axis was defined as the femoral $x$-axis (positive to the right) and the origin was defined as the midpoint between the centers of these posterior condylar spheres. The femoral $z$-axis was defined as the line perpendicular to the $x$-axis in a plane formed by the $x$-axis and a line connecting the femoral origin and the center of the femoral head (positive superiorly). The femoral $y$-axis was defined as the cross product of the $z$-axis and $x$-axis (positive anteriorly). For the tibia, the $z$-axis was defined by a line connecting the midpoint of the tibial eminences and those of the medial and lateral top of the talar dome (positive in the superior direction). The tibial $y$-axis (positive in the anterior direction) was the line perpendicular to the $z$-axis from the mediolateral center of the tibial insertion of the posterior cruciate ligament. The tibial $x$-axis was the cross-product of the $z$ - and $y$-axes (positive to the right)
(Bonferroni) when the data did not have a normal distribution. For comparison between AOA and FOA for the same subjects, the paired t-test was used when the data had a normal distribution and equal variance, and the Wilcoxon signed-rank test was used when the data had no normal distribution. The proportion of data on the basis of sex was evaluated by the Chi-square test. In all tests, the level of statistical significance was set at a $p$-value $<0.05$. Statistical analyses were performed using SPSS, version 26 (SPSS, Inc., Chicago, IL, USA). For the sample size calculation ( $\alpha$ error $=0.05,1-\beta$ error $=0.80$ ), a total number of 69 knees were required; this study included a total of 87 knees and was thus a sufficient sample size.

\section{Results}

A comparison of FOA of osteoarthritic with healthy knees is shown in Tables 2 and 3. In knee OA, we found that the femur was subjected to more medial inclination $(p<0.001)$ and the tibia to more lateral inclination in the coronal plane $(p=0.003)$. In the sagittal plane, the femur revealed more posterior inclination $(p=0.014)$ and the tibia revealed more anterior inclination $(p<0.001)$ in knee OA. In the transverse plane, the tibia revealed similar directionality $(p=0.945)$, whereas the femur exhibited more external rotation in knee $\mathrm{OA}(p=0.005)$.

Comparing measurements of TKA to the other conditions (Tables 2 and 3), it was found that both femur and tibia in TKA were aligned more vertically with respect
Fig. 5 Assessment parameters of the inclination of each bone and the lower leg alignment in FOA. $X w, Y w$, and $Z w=$ each axis of the world coordinate system, $F$ : medial femoral $=$ medial inclination, $F$ : lateral $=$ femoral lateral inclination, $T$ : medial $=$ tibial medial inclination, $T$ : lateral $=$ tibial lateral inclination, $F$ : anterior $=$ femoral anterior inclination, $F$ : posterior $=$ femoral posterior inclination, $T$ : anterior $=$ tibial anterior inclination, $T$ : posterior $=$ tibial posterior inclination

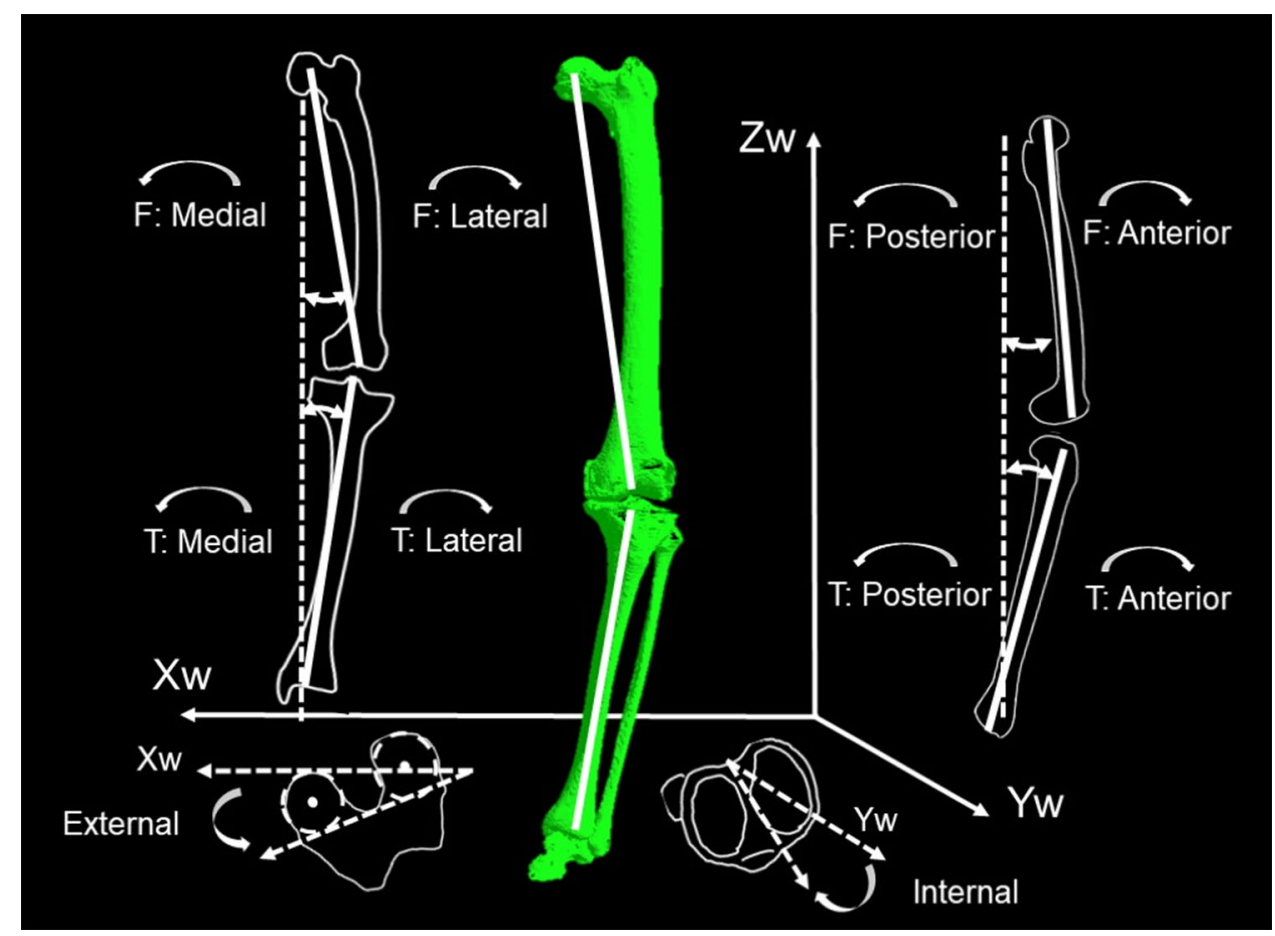


Fig. 6 Parameters of the difference angle between the anatomical plane and functional plane in each of the femur and tibia. $X w, Y w$, and $Z w=$ each axis of the world coordinate system; $X f$, $Y f$, and $Z f=$ each axis of the femoral coordinate system; $X t$, $Y t$, and $Z t=$ each axis of the tibial coordinate system

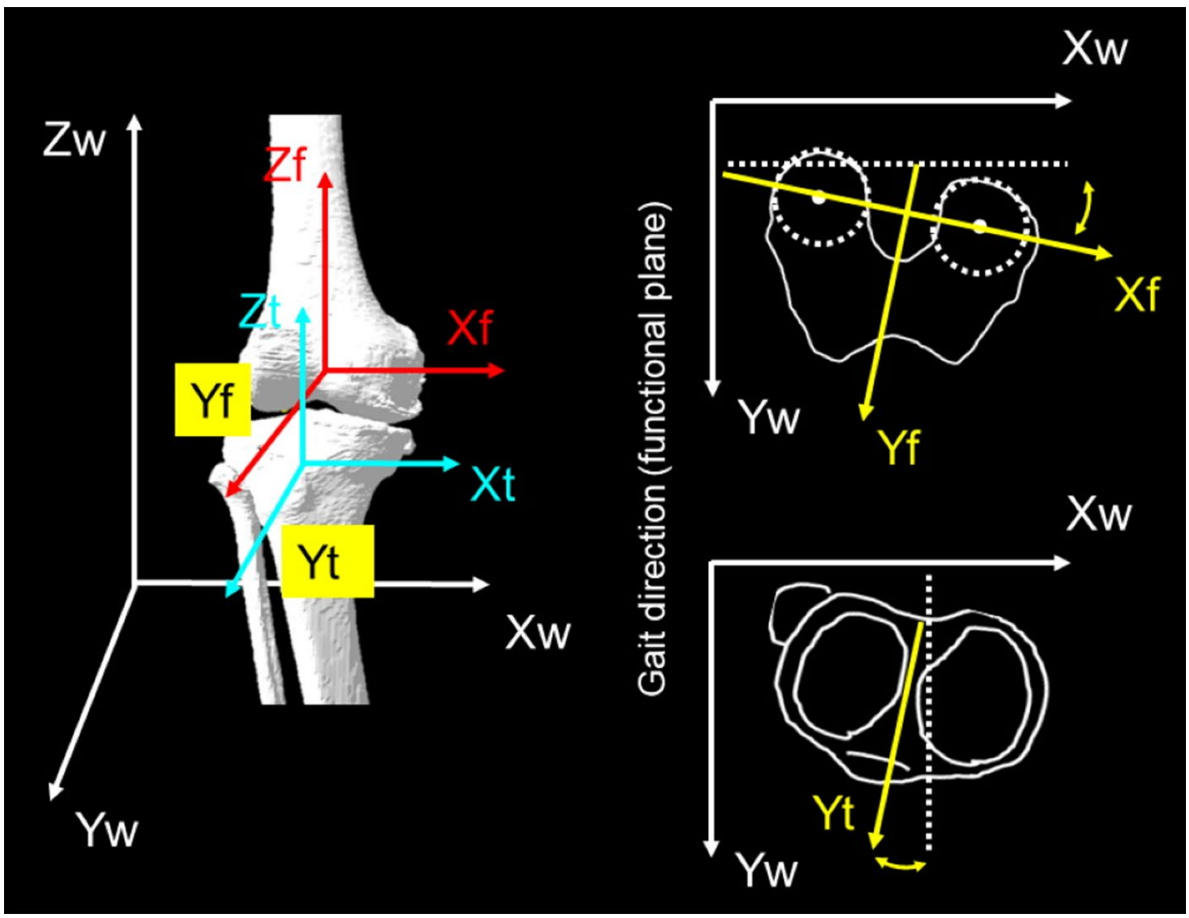

Table 2 Comparison of the alignment among the groups in one-way ANOVA

\begin{tabular}{|c|c|c|c|c|c|c|c|}
\hline \multirow[t]{2}{*}{ Variables } & \multicolumn{2}{|c|}{ Healthy $(n=24)$} & \multicolumn{2}{|c|}{ OA $(n=39)$} & \multicolumn{2}{|c|}{ TKA $(n=24)$} & \multirow[t]{2}{*}{$p$ value } \\
\hline & Mean & $95 \% \mathrm{CI}$ & Mean & $95 \% \mathrm{CI}$ & Mean & $95 \% \mathrm{CI}$ & \\
\hline Femoral coronal inclination (medial inclination: + ), ${ }^{\circ}$ & -1.6 & -2.4 to -0.9 & 2.5 & 1.4 to 3.5 & -1.3 & 2.1 to -0.5 & $<0.001^{*}$ \\
\hline Tibial coronal inclination (medial inclination: + ), ${ }^{\circ}$ & -5.0 & -5.8 to -4.2 & -7.7 & -9.0 to -6.3 & -4.2 & -5.6 to -2.7 & $<0.001 *$ \\
\hline Femoral sagittal inclination (anterior inclination: + ), ${ }^{\circ}$ & 4.6 & $3.1-6.2$ & 1.2 & -0.5 to 2.9 & 3.5 & $1.6-5.3$ & $0.013^{*}$ \\
\hline Tibial sagittal inclination (anterior inclination: + ), ${ }^{\circ}$ & 4.3 & $3.4-5.3$ & 8.6 & $7.1-10.1$ & 2.3 & $0.6-4.0$ & $<0.001 *$ \\
\hline Femoral transverse direction (external rotation: + ), ${ }^{\circ}$ & -6.8 & $-10.8--2.9$ & 1.5 & $-1.3-4.3$ & 5.5 & $1.6-9.3$ & $<0.001^{*}$ \\
\hline Tibial transverse direction (external rotation: + ), ${ }^{\circ}$ & -0.4 & $-3.3-2.4$ & 0.3 & $-2.8-3.3$ & 7.5 & $4.3-10.7$ & $0.001 *$ \\
\hline FOA: coronal alignment in HKA (larger: varus), ${ }^{\circ}$ & 183.5 & $182.6-184.4$ & 190.3 & $188.3-192.2$ & 182.3 & $181.5-183.1$ & $<0.001^{*}$ \\
\hline FOA: sagittal alignment in HKA (flexion: -), ${ }^{\circ}$ & 0.3 & $-1.9-2.5$ & -7.4 & $-10.2--4.6$ & 1.2 & $-1.6-4.0$ & $<0.001^{*}$ \\
\hline FOA: rotational alignment (larger: tibial internal rotation), ${ }^{\circ}$ & 83.4 & $80.2-87.0$ & 91.3 & $88.5-93.9$ & 88.0 & $85.4-90.6$ & $0.001 *$ \\
\hline AOA: coronal alignment in HKA (larger: varus), ${ }^{\circ}$ & 182.8 & $181.8-183.7$ & 190.5 & $188.3-192.7$ & 182.2 & $181.3-183.1$ & $<0.001^{*}$ \\
\hline AOA: sagittal alignment in HKA (flexion: -), ${ }^{\circ}$ & 0.9 & $-1.5-3.3$ & -7.2 & $-9.9--4.4$ & 1.0 & $-1.8-3.9$ & $<0.001 *$ \\
\hline AOA: rotational alignment (larger: tibial internal rotation), ${ }^{\circ}$ & 84.4 & $80.9-87.9$ & 92.1 & $88.0-96.1$ & 86.2 & $83.6-88.9$ & $<0.001^{*}$ \\
\hline $\begin{array}{l}\text { Femoral difference between the anatomical and functional } \\
\text { planes (external rotation: }+)^{\circ}\end{array}$ & -9.1 & $-12.8--5.5$ & -1.6 & $-4.6-1.4$ & 4.1 & $0.1-8.1$ & $<0.001 *$ \\
\hline $\begin{array}{l}\text { Tibial difference between the anatomical and functional } \\
\text { planes (external rotation: }+ \text { ) },^{\circ}\end{array}$ & 3.5 & $0.8-6.2$ & 3.3 & $-0.6-7.3$ & 8.9 & $5.3-12.5$ & 0.072 \\
\hline
\end{tabular}

$H K A$ Hip-knee-ankle angle, $F O A$ functionally oriented alignment, $A O A$ anatomically oriented alignment

*Significant difference $=p<0.05$, n.s. $=p>0.05$

to the ground than knee OA (femoral coronal inclination, $p<0.001$; tibial coronal inclination, $p<0.001$; tibial sagittal inclination, $p<0.001$ ); findings from TKA were statistically indistinguishable from those in the healthy controls (femoral coronal inclination, $p=0.890$; tibial coronal inclination, $p=0.385$; femoral sagittal inclination, $p=0.663$; tibial sagittal inclination, $p=0.467$ ). In the transverse plane, the tibia was more externally rotated in TKA than that in other conditions (healthy $v s$. TKA, $p=0.004$; OA $v s$. TKA, $p=0.003$ ). The femoral transverse direction in TKA was similar to that in knee OA $(p=0.559)$ but was different from that in the healthy subjects $(p<0.001)$. 
Table 3 Post-hoc test in oneway ANOVA

\begin{tabular}{lllc}
\hline$p$ values & Healthy vs OA & OA vs TKA & Healthy vs TKA \\
\hline Femoral coronal inclination & $<0.001^{*}$ & $<0.001^{*}$ & 0.890 \\
Tibial coronal inclination & $0.003^{*}$ & $<0.001^{*}$ & 0.385 \\
Femoral sagittal inclination & $0.014^{*}$ & 0.140 & 0.663 \\
Tibial sagittal inclination & $0.001^{*}$ & $<0.001^{*}$ & 0.467 \\
Femoral transverse direction & $0.005^{*}$ & 0.559 & $<0.001^{*}$ \\
Tibial transeverse direction & 0.945 & $0.003^{*}$ & $0.004^{*}$ \\
FOA: coronal alignment in HKA & $<0.001^{*}$ & $<0.001^{*}$ & 0.735 \\
FOA: sagittal alignment in HKA & $<0.001^{*}$ & $<0.001^{*}$ & 1.000 \\
FOA: rotational alignment & $0.001^{*}$ & 0.230 & 0.122 \\
AOA: coronal alignment in HKA & $<0.001^{*}$ & $<0.001^{*}$ & 1.000 \\
AOA: sagittal alignment in HKA & $<0.001^{*}$ & $<0.001^{*}$ & 0.997 \\
AOA: rotational alignment & $0.001^{*}$ & $0.013^{*}$ & 1.000 \\
Femoral difference between the anatomi- & $0.006^{*}$ & $0.049^{*}$ & $<0.001^{*}$ \\
cal and functional planes & & & 0.147 \\
Tibial difference between the anatomical & 0.997 & 0.081 & \\
and functional planes & & & \\
\hline
\end{tabular}

$H K A$ Hip-knee-ankle angle, $F O A$ functionally oriented alignment, $A O A$ anatomically oriented alignment *Significant difference $=p<0.05$, n.s. $=p>0.05$
Table 4 Comparison between FOA and AOA

\begin{tabular}{llll}
\hline$p$ values & Healthy & OA & TKA \\
\hline Coronal alignment in HKA & $0.006^{*}$ & 0.194 & 0.648 \\
Sagittal alignment in HKA & $0.001^{*}$ & 0.230 & 0.269 \\
Rotational alignment & 0.119 & $0.001^{*}$ & $0.021^{*}$ \\
\hline
\end{tabular}

HKA Hip-knee-ankle angle, FOA functionally oriented alignment, $A O A$ anatomically oriented alignment

$*$ Significant difference $=p<0.05$, n.s. $=p>0.05$

Regarding the difference between the anatomical and functional planes (Tables 2 and 3), the tibia revealed similar directionality, when compared healthy with osteoarthritic knees $(p=0.997)$, whereas the femur showed the larger deviation in the two planes for osteoarthritic knees than that for healthy knees. In TKA, the anatomical plane revealed a higher degree of external rotation relative to functional plane of the femur, compared to values determined for both healthy and osteoarthritic knees (healthy $v s$. TKA, $p<0.001$; OA $v s$. TKA, $p=0.049)$. For the tibia, TKA exhibited trend of the more external rotation in the anatomical plane relative to the functional plane than healthy and osteoarthritic knees, without the statistical differences (healthy $v s$. TKA, $p=0.147$; OA $v s$. TKA, $p=0.081$ ).

The comparisons between AOA and FOA demonstrated the differences in a part of data (coronal alignment: healthy, $p=0.006$; sagittal alignment: healthy, $p=0.001$; and rotational alignment: OA, $p=0.001$; TKA, $p=0.021$ ) (Table 4).

\section{Discussion}

The most important finding presented in this study is that the anatomical planes of the femur and tibia are either similar to or different from the functional planes, depending on the current condition of the subjects (i.e., healthy, knee OA, or TKA). The position of each bone relative to the direction of gravity also differed depending on the patients' diagnosis and surgical history. In addition, a part of FOA was different from AOA, in particular, for rotational alignment in knee $\mathrm{OA}$ and TKA.

To the best of our knowledge, this is the first study to assess FOA in weight-bearing conditions. One of the primary benefits of the FOA measurement is the possibility of evaluating alignment in the functional plane that reflects the direction of the toe during walking. Likewise, the use of FOA methods permit an evaluation of the inclination of each bone in the lower extremity relative to the direction of gravity which is not possible using AOA methodology. The use of FOA to evaluate the position of each bone relative to gravity and the differences between the functional and anatomic planes, provide critical information regarding pathology and kinematics in various disease states.

Using FOA methodology we found that, when compared with healthy knees, osteoarthritic knees have femur with more medial inclination, tibia with more lateral inclination in the coronal plane, and varus alignment as observed in the coronal alignment of the HKA. These results indicate that all bones in the lower extremity contributed to the varus alignment. In previous studies [4, 5], varus alignment of the advanced knee OA 
correlated with the degree of inclination of the medial compartment of the proximal tibia (MCT). Under weight-bearing conditions, the inclination of MCT was aligned parallel to the ground. With increasing degrees of varus misalignment, the moment arm for the gravitational force vector is increased, resulting in a significantly higher adduction moment than that in a normal knee [37]; the knee adduction moment gradually forces the tibia into the varus alignment $[38,39]$. At the hip joint, increased internal hip abduction moment during walking was associated with a reduced likelihood of medial tibiofemoral progression of OA [40]. In addition to knee adduction moment, hip abduction moment is also affected in advanced knee OA, resulting in more medial and lateral inclination of the femur and tibia, respectively.

With respect to sagittal alignment, the femur revealed more posterior inclination and the tibia revealed more anterior inclination in knee OA compared to the healthy controls. These results suggested that knee OA is associated with flexion contracture and the inclination of each bone is associated with an increase in knee flexion. An earlier study [32] reported that the larger posterior tibial slope was associated with higher knee flexion (sagittal alignment) in the standing position; it is likely that posterior tibial slope and sagittal alignment influence one another. As such, when humans stand upright and experience the gravitational force, the articular surface of the tibia should be parallel to the ground to permit efficient load-bearing [32]. The sagittal alignment of the knee has been clearly described as a compensatory mechanism for maintaining horizontal gaze. The primary aim of these efforts is to maintain the center of gravity centrally positioned over the feet [41] and to preserve a horizontal gaze [42]. The femoral angle is significantly associated with pelvic tilt which results in the aforementioned knee flexion [43]. Taken together, knee flexion in advanced OA is most likely the result of complicated connections among the compensatory mechanisms due to spinopelvic deformity, body constitution, neurological factors, and the impact of force generated by the tibial posterior slope, among others $[32,41-43]$.

The healthy and osteoarthritic knees showed similar rotational directions when comparing the functional plane and tibial anatomical plane, although we detected a large rotational deviation between the two planes in the femur. When humans walk in a forward direction, the functional planes defining the pelvis, hip, and knee joints should coincide [17, 18, 24]; extensor mechanisms, such as quadriceps femoris, should be maintained in the forward position during walking; the anatomical and functional planes of the tibia will then coincide. However, as shown here, the femur in OA patients demonstrated different associations between the two planes. Healthy knees exhibit the "screw-home" movement and demonstrate maximum internal rotation in association with terminal knee extension [33, 35, 36]. Osteoarthritic knees have various deformities, including the limited hip internal rotation (external rotation contracture), femoral neck anteversion, torsion in the femoral diaphysis, femorotibial rotational mismatch [17-20], and flexion contracture $[20,32]$. On a particular note, the limited hip internal rotation and the flexion contracture, as the knee joint externally rotates as knee flexes, is probably influenced $[35,36]$. As shown in this study, osteoarthritic knees demonstrated a greater degree of external rotation in a comparison between the anatomical and functional planes in the femur than did the knees of healthy subjects. From the viewpoint of the knee joint, the anatomical plane would ideally coincide with the functional plane during walking. The tibia showed a similar position between the anatomical and functional planes in healthy and osteoarthritic knees. The results presented here suggest that, in both healthy and advanced osteoarthritic knees, mechanisms promoting the coincidence between the gait direction and bony anatomical plane were functioning in the tibia, not in the femur.

Coronal and sagittal inclination in TKA, compared to those with knee OA, included the femur and tibia aligned more vertically to the ground, and limb alignment after TKA reverted more closely to the healthy state. TKA could realize joint line's parallel phenomenon relative to gravity by the component position perpendicular to the mechanical axis. In the transverse plane, the femur in TKA knees rotated more externally, relative to the gait direction than that in healthy knees, while it was not significantly different between TKA and OA. Subjects with knee OA have shown extra-articular deformities such as limited hip internal rotation [17-20]. In gait, most subjects with advanced knee OA demonstrated the toe out gait due to the limited hip internal rotation (external rotation contracture) in this study [17]. Although TKA has the potential to correct rotational alignment of the lower extremity [8, 19], results of this study (femoral external rotation in FOA) indicate that TKA could not perfectly correct the overall alignment in the transverse plane with the extra-articular deformities in knee OA. The tibia in TKA knees rotated more externally, relative to the gait direction in the transverse plane than that in healthy and osteoarthritic knees. The residual postoperative femoral external rotation due to the uncorrectable extra-articular femoral deformities (e.g., hip external rotation) [17-20] and structural constraint between the component and insert in the medial pivot implants might contribute to tibial external rotation in FOA; tibial transverse direction is likely to be determined by femoral transverse direction after arthroplasties. In TKA, mechanisms promoting the coincidence between the gait direction and the bony anatomical plane are assumed to mainly function in the femur by the implant constraint and the extra-articular femoral deformities. This study showed that the femoral rotational position in TKA was of importance. The implant used was medial pivot 
type inducing "medial pivot motion" to provide stability in the anterior and posterior directions [44, 45]. The authors applied the three-dimensional preoperative planning and the unique jig, reproducing its planning for highly accurate surgery [46]. Especially, the femoral rotational alignment, as a reference of the surgical epicondylar axis, can be shown as high accuracy by using the jig [46]. However, as the extraarticular deformities could not be corrected in TKA, the surgeons, at least, should pay attention to achieve femoral rotational position as accurate as possible. Besides, since the constraint of the femoral implant forces determine the tibial rotation, the rotational mismatch between the femoral and tibial implants $[22,23]$ should be minimized to avoid serious adverse effects.

This study proved that a part of FOA was different from AOA, especially, in rotational alignment in knee OA and TKA. Although the differences were not large, but they may explain eccentricity and overloading at the knee joint, leading to the onset and progression of knee $\mathrm{OA}$ and persistence of serious problems even after TKA [22, 23]. If the AOA and FOA methodologies are presented by a common X-ray, the radiographs in the knee forward view and natural standing position view may correspond to AOA and FOA, respectively. In actual clinical setting, the two X-rays might have the potential to solve clinical problems. The differences in the radiographs can suggest several possible reasons such as the bony deformity (e.g., torsional deformity), the rotational change between the bones, and the technical error in TKA. For example, even if the X-ray in the knee forward view (AOA) is within the acceptance range, it may not mean the acceptable alignment for the function of the lower extremity (FOA), which means discordance between the gait direction and the anteroposterior axis of the implant, possibly causing eccentricity and overloading on the implants.

There were several limitations in this study. Firstly, the images analyzed were not of a single leg in walking position, but of both legs standing in a static position. While the single-leg standing position may better reflect the posture during walking, but the elderly subjects were not able to maintain this position during biplanar radiography, and any movement during the procedure would reduce the accuracy of the images. Secondly, this study could not perfectly assess the lower extremity alignment in the stance phase during gait. Ideally, the dynamic assessment, such as a motion capture system, is the best way to investigate the alignment in the gait, but the dynamic assessment cannot ensure enough accuracy to identify the rotational parameters. The method in the present study could ensure adequate accuracy within the range of one degree for the rotational parameters and could clarify one of the alignments at a certain moment during the stance phase. Thirdly, the demographic data (age, BMI, toe angle, and sex) had differences among the groups, which might lead to the bias, but the differences in age and BMI were not large. Also, a part of the data was collected from both the knees (right and left sides) of the same subjects and was not independent. However, since independently evaluated the right and left sides for toe angles directly affecting the lower extremity alignment, the confounding effects caused by pairs of knees were minimized. Regarding sex, most subjects with knee OA and TKA were females. Since the male and female data is assumed to have different results, further study is required to investigate the data with no differences in the background factors, such as sex.

\section{Conclusions}

This is the first study to define the FOA and to examine bones of lower extremities under weight-bearing conditions for healthy, knee OA, and TKA subjects. The association between the anatomical and functional planes, and the position of each bone relative to the direction of gravity in FOA were different, depending on the current condition of subjects. This study suggests that, in both healthy and advanced osteoarthritic knees, mechanisms promoting the coincidence between gait direction and bony anatomical plane function in tibia, not femur. In TKA knees, these mechanisms mainly function in femur by the implant constraints and extra-articular femoral deformities. Additionally, the AOA and FOA methodology showed the partial differences, implying that the differences in the position during radiography could help to solve the clinical problems. For clinical relevance, the FOA concept may potentially clarify one of the causative factors of the onset and progression of knee OA and serious adverse problems after TKA.

Acknowledgements The authors would like to thank all stuff members in Radiology and Rehabilitation Departments in Niigata Medical Center.

Authors Contributions Conception and design: Dr. ST. Provision of study materials or patients: Dr. ST. Collection of data and analysis and interpretation of the data: Drs. ST, MT, KR, TY. Technical support for the system of extremity alignment assessment system: Dr. ST. Drafting of the article: Drs. ST, MT. Final approval of the article: Drs. ST, MT, KR, TY.

Funding This study received KAKENHI from grants-in-aid for scientific research in Japan society for the promotion of science.

Data Availability References see PubMed.gov.

\section{Compliance with Ethical Standards}

Conflict of Interest All authors declare that they have no conflict of interest. 
Ethics Approval and Consent to Participate The study was approved by the Institutional Review Board in Niigata Medical Center. Appropriate consents, permissions and releases were obtained.

Informed Consent All presentations had consent to publish.

Open Access This article is licensed under a Creative Commons Attribution 4.0 International License, which permits use, sharing, adaptation, distribution and reproduction in any medium or format, as long as you give appropriate credit to the original author(s) and the source, provide a link to the Creative Commons licence, and indicate if changes were made. The images or other third party material in this article are included in the article's Creative Commons licence, unless indicated otherwise in a credit line to the material. If material is not included in the article's Creative Commons licence and your intended use is not permitted by statutory regulation or exceeds the permitted use, you will need to obtain permission directly from the copyright holder. To view a copy of this licence, visit http://creativecommons.org/licenses/by/4.0/.

\section{References}

1. Cicuttini, F., Wluka, A., Hankin, J., \& Wang, Y. (2004). Longitudinal study of the relationship between knee angle and tibiofemoral cartilage volume in subjects with knee osteoarthritis. Rheumatology (Oxford), 43(3), 321-324.

2. Higano, Y., Hayami, T., Omori, G., Koga, Y., Endo, K., \& Endo, N. (2016). The varus alignment and morphologic alterations of proximal tibia affect the onset of medial knee osteoarthritis in rural Japanese women: case control study from the longitudinal evaluation of Matsudai Knee Osteoarthritis Survey. J Orthop Sci, 21(2), 166-171.

3. Matsumoto, T., Hashimura, M., Takayama, K., Ishida, K., Kawakami, Y., Matsuzaki, T., et al. (2015). A radiographic analysis of alignment of the lower extremities-initiation and progression of varus-type knee osteoarthritis. Osteoarthritis Cartilage, 23(2), 217-223.

4. Mochizuki, T., Koga, Y., Tanifuji, O., Sato, T., Watanabe, S., Koga, H., et al. (2019). Effect on inclined medial proximal tibial articulation for varus alignment in advanced knee osteoarthritis. Journal of Experimental Orthopaedics, 6, 14. https://doi. org/10.1186/s40634-019-0180-x.

5. Mochizuki, T., Koga, Y., Mori, T., Nishino, K., Kobayashi, K., Tanifuji, O., et al. (2019). Articular surface of the medial proximal tibia is aligned parallel to the ground in three-dimensional space under weight-bearing conditions in healthy and varus osteoarthritic knees. Knee Surgery, Sports Traumatology, Arthroscopy. https://doi.org/10.1007/s00167-019-05829-0.

6. Matsuda, S., Miura, H., Nagamine, R., Urabe, K., Hirata, G., \& Iwamoto, Y. (2001). Effect of femoral and tibial component position on patellar tracking following total knee arthroplasty: 10-year follow-up of Miller-Galante I knees. American Journal of Knee Surgery, 14(3), 152-156.

7. Nicoll, D., \& Rowley, D. (2010). Internal rotational error of the tibial component is a major cause of pain after total knee replacement. Journal of Bone and Joint Surgery. British Volume, 92(9), $1238-1244$.

8. Watanabe, S., Sato, T., Omori, G., Koga, Y., \& Endo, N. (2014). Change in tibiofemoral rotational alignment during total knee arthroplasty. J Orthop Sci, 19(4), 571-578.

9. Ariumi, A., Sato, T., Kobayashi, K., Koga, Y., Omori, G., Minato, I., et al. (2010). Three-dimensional lower extremity alignment in the weight-bearing standing position in healthy elderly subjects. J Orthop Sci, 15(1), 64-70.

10. Fujii, T., Sato, T., Ariumi, A., Omori, G., Koga, Y., \& Endo, N. (2020). A comparative study of weight-bearing and non-weightbearing 3-dimensional lower extremity alignment in knee osteoarthritis. Journal of Orthopaedic Science. https://doi.org/10.1016/j. jos.2019.11.012.

11. Katsumi, R., Mochizuki, T., Sato, T., Kobayashi, K., Watanabe, S., Tanifuji, O., et al. (2018). Contribution of sex and body constitution to three-dimensional lower extremity alignment for healthy, elderly, non-obese humans in a Japanese population. Journal of Experimental Orthopaedics, 5, 32. https://doi.org/10.1186/s4063 4-018-0147-3.

12. Asano, T., Akagi, M., \& Nakamura, T. (2005). The functional flexion-extension axis of the knee corresponds to the surgical epicondylar axis: in vivo analysis using a biplanar image matching technique. Journal of Arthroplasty, 20(8), 1060-1067.

13. Churchill, D. L., Incavo, S. J., Johnson, C. C., \& Beynnon, B. D. (1998). The transepicondylar axis approximates the optimal flexion axis of the knee. Clinical Orthopaedics and Related Research, $356,111-118$

14. Hollister, A. M., Jatana, A., Singh, A. K., Sullivan, W. W., \& Lupichuk, A. G. (1993). The axes of rotation of the knee. Clinical Orthopaedics and Related Research, 290, 259-268.

15. Sato, T., Koga, Y., \& Omori, G. (2004). Three-dimensional lower extremity alignment assessment system: application to evaluation of component position after total knee arthroplasty. Journal of Arthroplasty, 19(5), 620-628.

16. Sato, T., Koga, Y., Sobue, T., Omori, G., Tanabe, Y., \& Sakamoto, M. (2007). Quantitative 3-dimensional analysis of preoperative and postoperative joint lines in total kneearthroplasty: a new concept for evaluation of component alignment. Journal of Arthroplasty, 22(4), 560-568.

17. Mochizuki, T., Tanifuji, O., Koga, Y., Sato, T., Kobayashi, K., Nishino, K., et al. (2017). Sex differences in femoral deformity determined using three-dimensional assessment for osteoarthritic knees. Knee Surgery, Sports Traumatology, Arthroscopy, 25(2), 468-476.

18. Mochizuki, T., Tanifuji, O., Koga, Y., Hata, R., Mori, T., Nishino, K., et al. (2017). External torsion in a proximal tibia and internal torsion in a distal tibia occur independently in varus osteoarthritic knees compared to healthy knees. J Orthop Sci, 22(3), 501-505.

19. Mochizuki, T., Sato, T., Tanifuji, O., Watanabe, S., Kobayashi, K., \& Endo, N. (2018). Extrinsic factors as component positions to bone and intrinsic factors affecting postoperative rotational limb alignment in total knee arthroplasty. Journal of Arthroplasty, 33(7), 2100-2110.

20. Koga, Y. (2008). Osteoarthritic of the knee Epidemiology, biomechanics, and conservative treatment, Chapter 2 [In Japansese] (pp. 41-65). Japan: Nankodo.

21. Nishino, K., Omori, G., Koga, Y., Kobayashi, K., Sakamoto, M., Tanabe, Y., et al. (2015). Three-dimensional dynamic analysis of knee joint during gait in medial knee osteoarthritis using loading axis of knee. Gait Posture, 42(2), 127-132.

22. Berger, R. A., Crossett, L. S., Jacobs, J. J., \& Rubash, H. E. (1998), Malrotation causing patellofemoral complications after total knee arthroplasty. Clinical Orthopaedics and Related Research, 356, 144-153.

23. Incavo, S. J., Wild, J. J., Coughlin, K. M., \& Beynnon, B. D. (2007). Early revision for component malrotation in total knee arthroplasty. Clinical Orthopaedics and Related Research, 458, 131-136.

24. Imai, N., Ito, T., Takahashi, Y., Horigome, Y., Suda, K., Miyasaka, D., et al. (2013). In vivo relationship between the clinical epicondylar axis and the anterior pelvic plane in normal subjects. Journal of Biomedical Science, 6, 863-868. 
25. Kellgren, J. H., \& Lawrence, J. S. (1957). Radiological assessment of osteo-arthrosis. Annals of the Rheumatic Diseases, 16, 494-502.

26. Akagi, M., Mori, S., Nishimura, S., Nishimura, A., Asano, T., \& Hamanishi, C. (2005). Variability of extraarticular tibial rotation references for total knee arthroplasty. Clinical Orthopaedics and Related Research, 436, 172-176.

27. Kobayashi, K., Sakamoto, M., Tanabe, Y., Ariumi, A., Sato, T., Omori, G., et al. (2009). Automated image registration for assessing three-dimensional alignment of entire lower extremity and implant position using bi-plane radiography. Journal of Biomechanics, 42(6), 2818-2822.

28. Mochizuki, T., Sato, T., Tanifuji, O., Kobayashi, K., Koga, Y., Yamagiwa, H., et al. (2013). In vivo pre- and postoperative threedimensional knee kinematics in unicompartmental knee arthroplasty. Journal of Orthopaedic Science, 18(1), 54-60.

29. Mochizuki, T., Sato, T., Blaha, J. D., Tanifuji, O., Kobayashi, K., Yamagiwa, H., et al. (2014). Kinematics of the knee after unicompartmental arthroplasty is not the same as normal and is similar to the kinematics of the knee with osteoarthritis. Knee Surgery, Sports Traumatology, Arthroscopy, 22(8), 1911-1917.

30. Mochizuki, T., Sato, T., Blaha, J. D., Tanifuji, O., Kobayashi, K., Yamagiwa, H., et al. (2014). The clinical epicondylar axis is not the functional flexion axis of the human knee. Journal of Orthopaedic Science, 19(3), 451-456.

31. Mochizuki, T., Sato, T., Tanifuji, O., Kobayashi, K., Yamagiwa, H., Watanabe, S., et al. (2015). Unicompartmental knee arthroplasty cannot restore the functional flexion axis of a living knee to normal. Knee Surgery, Sports Traumatology, Arthroscopy, 23(12), 3736-3742.

32. Mochizuki, T., Tanifuji, O., Koga, Y., Sato, T., Kobayashi, K., Watanabe, S., et al. (2018). Correlation between posterior tibial slope and sagittal alignment under weight-bearing conditions in osteoarthritic knees. PLoS One, 13, e0202488. https://doi. org/10.1371/journal.pone.0202488.

33. Murayama, T., Sato, T., Watanabe, S., Kobayashi, K., Tanifuji, O., Mochizuki, T., et al. (2016). Three-dimensional in vivo dynamic motion analysis of anterior cruciate ligament-deficient knees during squatting using geometric center axis of the femur. Journal of Orthopaedic Science, 21(2), 159-165.

34. Takagi, S., Sato, T., Watanabe, S., Tanifuji, O., Mochizuki, T., Omori, G., et al. (2018). Alignment in the transverse plane, but not sagittal or coronal plane, affects the risk of recurrent patella dislocation. Knee Surgery, Sports Traumatology, Arthroscopy, 26(7), 2891-2898.
35. Tanifuji, O., Sato, T., Kobayashi, K., Mochizuki, T., Koga, Y., Yamagiwa, H., et al. (2011). Three-dimensional in vivo motion analysis of normal knees using single-plane fluoroscopy. Journal of Orthopaedic Science, 16(6), 710-718.

36. Tanifuji, O., Sato, T., Kobayashi, K., Mochizuki, T., Koga, Y., Yamagiwa, H., et al. (2013). Three-dimensional in vivo motion analysis of normal knees employing transepicondylar axis as an evaluation parameter. Knee Surgery, Sports Traumatology, Arthroscopy, 21(10), 2301-2308.

37. Schipplein, O. D., \& Andriacchi, T. P. (1991). Interaction between active and passive knee stabilizers during level walking. Journal of Orthopaedic Research, 9(1), 113-119.

38. Birmingham, T. B., Hunt, M. A., Jones, I. C., Jenkyn, T. R., \& Giffin, J. R. (2007). Test-retest reliability of the peak knee adduction moment during walking in patients with medial compartment knee osteoarthritis. Arthritis and Rheumatism, 57(6), 1012-1017.

39. Zhao, D., Banks, S. A., Mitchell, K. H., Lima, D. D., Colwell, C. W., \& Fregly, B. J. (2007). Correlation between the knee adduction torque and medial contact force for a variety of gait patterns. Journal of Orthopaedic Research, 25(6), 789-797.

40. Wang, Z., Molenaar, P. C., Challis, J. H., Jordan, K., \& Newell, K. M. (2014). Visual information and multijoint coordination patterns in one-leg stance. Gait Posture, 39(3), 909-914.

41. Jalai, C. M., Diebo, B. G., Cruz, D. L., Poorman, G. W., Vira, S., Buckland, A. J., et al. (2017). The impact of obesity on compensatory mechanisms in response to progressive sagittal malalignment Cyrus M. Spine J, 17(5), 681-688.

42. Hasegawa, K., Okamoto, M., Hatsushikano, S., Shimoda, H., Ono, M., \& Watanabe, K. (2016). Normative values of spino-pelvic sagittal alignment, balance, age, and health-related quality of life in a cohort of healthy adult subjects. European Spine Journal, 25(11), 3675-3686.

43. Lee, C. S., Park, S. J., Chung, S. S., \& Lee, K. H. (2013). The effect of Simulated knee flexion on sagittal alignment: novel interpretation of spinopelvic alignment. European Spine Journal, 22(5), 1059-1065.

44. Blaha, J. D. (2002). A medial pivot geometry. Orthpedisc, 25, 963-964.

45. Blaha, J. D. (2004). The rationale for a total knee implant that confers anteroposterior stability throughout range of motion. Journal of Arthroplasty, 19, 22-26.

46. Sato T, Kobayashi K, Tanifuji O, Omori G, Koga Y. (2010). Clinical accuracy of component alignment using three-dimensionally planned and controlled insertion of im alignment rod in TKA. Orthopaedic Research Society, Presentation. 